\title{
Interprofessional Cooperation: An Interventional Study Among Saudi Healthcare Teaching Staff at King Saud University
}

This article was published in the following Dove Press journal: Journal of Multidisciplinary Healthcare

\author{
Adel S Bashatah (D) \\ Khalid A Al-Ahmary ${ }^{2}$ \\ Mohamed Al Arifi ${ }^{3}$ \\ Yousif A Asiri ${ }^{3}$ \\ Yazed AIRuthia $\mathbb{1 D}^{3}$ \\ Ahmed Sayed Metwally ${ }^{4}$ \\ Christine A King ${ }^{5}$ \\ Brett Williams $\mathbb{D}^{5}$ \\ 'Department of Nursing Administration \\ \& Education, College of Nursing, King \\ Saud University, Riyadh, Saudi Arabia; \\ ${ }^{2}$ College of Public Health, King Saud Bin \\ Abdul-Aziz University for Health Science, \\ Jeddah, Saudi Arabia; ${ }^{3}$ Department of \\ Clinical Pharmacy, College of Pharmacy, \\ King Saud University, Riyadh, Saudi \\ Arabia; ${ }^{4}$ Department of Mathematics, \\ College of Science, King Saud University, \\ Riyadh, Saudi Arabia; ${ }^{5}$ Department of \\ Paramedicine, Monash University, \\ Frankston, VIC, Australia
}

Background: Collaborative interprofessional practice improves health outcomes. Interprofessional education (IPE) is essential in improving this collaboration and the quality of care. Although the majority of IPE research focuses on students, the delivery of IPE requires multiple levels of support within educational institutions, particularly teaching staff that are positive about and advocate for IPE. This study explored the attitudes of teaching staff towards interprofessional collaboration across a range of professions in Health at King Saud University, Saudi Arabia.

Methods: A pre-test post-test design was used with 53 teaching staff from the Health Colleges, King Saud University, before and after an interprofessional development workshop. A 12-item, 3-subscale version of the IEPS was used to evaluate changes in the 3-subscales "competency and autonomy", "perceived need for cooperation" and "perception of actual cooperation".

Results: This study involved teaching staff from medicine, nursing, pharmacy, dentistry, applied medical science and emergency medical services. Results showed positive attitudes towards IPE, including competency and autonomy, the need for cooperation, and the perception of actual cooperation. The analysis also showed a statistically significant effect of subscale 1 (competency and autonomy) was produced between the pre- and postworkshop training.

Conclusion: Interprofessional collaboration across the Health Colleges is an essential component of IPE, just as IPE is an integral component of interprofessional collaborative practice. The findings provided a baseline, as well as an incentive, for further development in IPE, from policy through to practice, across the Health Colleges. Findings also showed teaching staff having a positive attitude towards interprofessional collaboration. Further research is needed on tools for measuring IPC across university hierarchies and disciplines, as well as on enablers of IPE (and not just barriers).

Keywords: interdisciplinary communication, interprofessional collaboration, interprofessional education, health occupations, Saudi Arabia

\section{Introduction}

There is sufficient evidence to state that collaborative interprofessional practice improves health outcomes. ${ }^{1}$ With this recognition, two specific concepts have been brought to the fore, namely, Interprofessional collaboration (IPC) and Interprofessional education (IPE). The most recognised definition of IPC is when

Multiple health workers from different professional backgrounds work together with patients, families, carers and communities to deliver the highest quality care. ${ }^{1}$
Correspondence: Adel S Bashatah Department of Nursing Education \& Administration, College of Nursing, King Saud University, Riyadh, Saudi Arabia Email abashatah@ksu.edu.sa 
IPE, on the other hand, occurs when two or more professions learn with, from and about each other to improve collaboration and the quality of care. ${ }^{2}$ Interprofessional Education (IPE) has been proposed to create a collaborative practiceready workforce better equipped to address challenges faced by health systems worldwide and improve health outcomes. ${ }^{1}$ The goal of IPE is to reduce communication errors, enhance knowledge and perception of others' roles, and strengthen the coordination of health-care goals within a team. ${ }^{3}$ This has been shown to improve patient outcomes, adherence to guidelines regarding standards of care, and patient satisfaction; ${ }^{3}$ improve patient outcomes and reduce medical errors; ${ }^{4}$ and support the development of a pool of collaborative-practice ready professionals for improved health-care team development. ${ }^{5}$ Interprofessional education (IPE) is now widely accepted as being critical to an effective healthcare system. ${ }^{6}$ This study aims to contribute to the literature by exploring IPC in a region where there are few IPC and IPE studies and uses a tool that has shown promise in measuring attitudes to IPC but has had limited use. In addition, the study also explores interventions to enhance attitudes towards IPC and the approach previous studies have taken to address IPC and IPE (ie, addressing barriers or enablers).

\section{Background}

By far, the majority of IPE focuses on students, as does IPE research. Studies of students' attitudes towards IPE are seen in a wide range of disciplines such as medicine, nutrition, paramedicine, dentistry, nursing, pharmacy, occupational therapy, podiatry, speech therapy and allied health $^{3,7,8}$ with examples from across the globe. 9,10 Health professional educators and institutions are expected to ensure graduates understand the role of the health professions ${ }^{11}$ and are competent in their discipline and also in working collaboratively in health-care teams. ${ }^{12}$ The logical follow on being improved communication and teamwork leading to improved patient safety. Many would say that it should be a top priority to assess interprofessionalism among students to ensure effective outcomes of practice experiences and other educational activities. $^{11}$

Several studies have taken place in Saudi Arabia to look at IPE and student preparedness. For example, ${ }^{11}$ investigated pharmacy students' interprofessional perceptions toward pharmacy professions in Saudi Arabia; ${ }^{13}$ discusses how the Essential Skills in Health Professions Education (ESHPE) program was designed to promote learning in interprofessional teams (from 13 health institutions) for the Faculty of Health Sciences, Imam Abdulrahman Bin Faisal University, Saudi Arabia; and Fallatah $^{4}$ addresses the possibility of introducing IPE into health profession education in Saudi Arabia to support Saudi Vision 2030 of improving all aspects of the Saudi population's wellbeing and development. This vision highlights the recognition by the Saudi government that providing optimal care in health-care institutions requires health-care providers from different services to collaborate and interact or engage in IPC. IPC and IPE, however, is a relatively new concept in Saudi Arabia and other Middle Eastern countries, compared to the Western world. ${ }^{4}$

Judge et $\mathrm{al}^{5}$ describe the traditional approach to IPE as having "little to no interdisciplinary exposure until students enter their clinical training component". This has fortunately changed, particularly over the past decade, where IPE is being seen as an overall curriculum; with many suggestions on how this can be done. ${ }^{14}$ For example, El-Awaisi et al ${ }^{15}$ propose a 12-step strategy which is designed to focus on 12 key areas of IPE that will enhance the teaching of students and practitioners as they strive to become competent collaborators. Chen et $\mathrm{al}^{16}$ report on a curriculum with incorporated interprofessional sessions in blended modes, where sessions address the Interprofessional Education Collaborative (IPEC) competency domains: interprofessional collaborative practice, roles and responsibilities, interprofessional communication, conflict management, and leadership/membership. Teaching strategies included in these sessions include expert interviews, videos, reflections, pair-share, role play, and group debrief. ${ }^{16}$

Although the majority of IPE research focuses on students, the delivery of IPE requires multiple levels of support within educational institutions, from administration to faculty. ${ }^{17}$ For this reason, it makes sense to also research the attitudes of faculty staff to IPC and IPE so that interventions at the institutional level can be developed to support IPE delivery to students. Dallaghan et al ${ }^{17}$ note the benefits to educators that embrace IPE as increased networking and relationship opportunities, as well as new insights. They also note that the increased logistics and time required to implement IPE can negatively affect faculty and educator attitudes towards IPE and potentially undermine the intentions of IPE activities, with staff becoming reluctant to participate and students picking up on subtle negative cues. In a review of 21 evaluations of IPE, Hammick et $\mathrm{al}^{18}$ found that staff development is a crucial influence on the effectiveness of IPE for learners and that although IPE is often received positively by learners, it is less able to influence attitudes 
and perceptions towards others who deliver IPE positively. These findings highlight the need for assessing faculty attitudes towards IPE; however, Dallaghan et $\mathrm{al}^{17}$ suggests that most IPE research focuses on needs, outcomes and process of IPE, and research on faculty attitudes is lacking.

Students' attitudes, which are affected by various factors, have been recognised as the most important predictors of successful implementation of interprofessional education. ${ }^{19}$ Multiple scales and instruments have been developed to measure students' attitudes, but perhaps the most widely used are the Interdisciplinary Education Perception Scale (IEPS) ${ }^{20}$ and the Readiness for Interprofessional Learning Scale (RIPLS). Although research groups tend to favour one or the other, the Interprofessional Learning Group (IPL) at Glasgow Caledonian University has been using both the IEPS and RIPLS to monitor changes in attitudes and perceptions of undergraduate students from eight different health and social care programmes. ${ }^{21}$ Although the IEPS has been widely used to assess student attitudes, several studies use this tool to measure teaching staff and faculty attitudes. ${ }^{22,23}$ Also, multiple researchers have published alternative factor structures for the IEPS. ${ }^{20}$

Williams et $\mathrm{al}^{6}$ explain that even though the use of IEPS is widespread, its reliability and validity are often undermined when used in countries where English is a second language. This is supported in a study by Bonello et $\mathrm{al}^{7}$ that found that national culture influences IPE and what works in one culture may not work in another. For this reason, several studies have validated the use of IEPS in non-English speaking counties. For example, Williams et $\mathrm{al}^{6}$ translated the IEPS into Swedish. They validated the psychometric properties by using it with 164 medical and nursing, occupational therapy and physiotherapy students on clinical placements in Stockholm. The Serbian version of the Readiness for Interprofessional Learning Scale has also been translated and proven to be reliable and valid for the "teamwork, collaboration and shared learning" subscale.

In contrast, the "role and responsibilities" subscale showed lower stability. The results of this study revealed positive students' attitudes towards interprofessional learning. ${ }^{19}$ As Barr $^{14}$ notes, the findings regarding the impact of IPE cannot be transferred from a developed to a developing country without considering context and cultural differences.
This study explored the attitudes of teaching staff towards interprofessional collaboration across a range of professions in Health at King Saud University, Saudi Arabia. It also assessed any changes in attitudes because of an intervention-based interprofessional development workshop with these teaching staff. To add to the research on IEPS, this study also used the 12-item IEPS as suggested by McFadyen et $\mathrm{al}^{21}$ where Williams et $\mathrm{al}^{24}$ was unable to find any published work using this alternative factor IEPS and subsequently used this alternative with paramedic students. We were also unable to find any other examples of using this tool with students or teaching staff at the time of our study.

\section{Method \\ Design}

A repeated measures design was used with 53 teaching staff from the Health Colleges, King Saud University, before and after they attended an interprofessional development workshop.

These pre-workshop and post-workshop evaluations (collected at four weeks) enabled the assessment of both, the level of, and changes in, competency and autonomy, perceived need for cooperation, and perception of actual cooperation. Participants were invited to attend the workshop as part of their annual and ongoing professional development programs.

\section{Procedures}

The workshop aimed to promote greater understanding of Interprofessional Education and Practice (IPE\&P) and was facilitated by an IPE expert from the United States. It was an eight-hour training course from ( 9 am-5 pm) held at King Saud University (KSU) training venues. Participants included teaching staff from the Medical and Health faculty and practitioners in five specialties, Medicine, Nursing, Dentistry, Pharmacy, and Applied Medical Sciences that has some specialties such as Physical Therapy, Nutrition and Emergency Medical Services.

The workshop focused on basic concepts of IPE\&P, exploring the different models of IPE\&P in terms of didactic and clinical settings. Also, the workshop examined different types of curriculum development steps related to IPE\&P, various measurement tools and the organisational structures at academic institutions that engage the IPE\&P curriculum and practice in academic and clinical settings with the support of stakeholders and 
institutions' leaders. Other elements of the workshop included barriers and obstacles to IPE\&P, professional identity and roles of different health professions and solutions for better integration into health curricula programs. The workshop was undertaken using both didactic and small group teaching strategies. At the end of the workshop, participants were given a certificate of attendance with approved CME hours accredited by KSUDeanship of Skills Development.

\section{Instrumentation}

This study used the 12-item, 3-subscale version of the IEPS developed by McFadyen et al. ${ }^{21}$ The IEPS is one of the most commonly used questionnaires to measure agreement towards IPE and IPC. ${ }^{25}$ This version uses a 6-point Likert scale for responses $(1=$ strongly disagree and $6=$ strongly agree). The 3 -subscales are competency and autonomy (Factor 1 [range 5-30]), perceived need for cooperation (Factor 2 [range 2-12]), and perception of actual cooperation (Factor 3 [range 5-30]). This version is a modified and more reliable version of the 18-item, 4-subscale version, developed by Luecht et al. ${ }^{26}$ Demographic data were also collected from participants, including age, gender, college, profession and previous exposure to interprofessional training.

\section{Data Analysis}

The Statistical Package for Social Sciences (SPSS) version (23) was used for data storage and tabulation of the quantitative data. Descriptive statistics were used (mean and standard deviation) to describe the participant demographic information. All tests were two-tailed with the results considered statistically significant if the $p$-value is $<0.05$. Data were analysed for changes in competency and autonomy, perceived need for cooperation, and perception of actual cooperation for overall participants using a repeated measures design. Additional data in terms of interprofessional educational experience were also collected and analysed.

\section{Ethics}

The ethical approval was obtained from the King Saud University Institutional Review Board office, Health Sciences Colleges Research on Human Subject, which is located in the College of Medicine at King Saud University. The approval research number (E-18-3610). Informed written consent was obtained from all participants. All procedures performed in this study were following the principles stated in the Declaration of Helsinki, ethical standards of the institutional and national research committee.

\section{Results}

\section{Participants}

The majority of participants were male; $\mathrm{n}=30$ (56.6\%) while females were $n=23(43.4 \%)$, which is consistent with the broader profile of university teaching staff within Saudi Arabia. The age distribution showed the majority of participants being between 33 and 60 years old; the mean age $m=$ 45.17 years, $S D=8.05$. The majority of participants were assistant professors, $\mathrm{n}=35$ (66\%); compared with professors, $\mathrm{n}=11(20.8 \%)$ and associate professors, $\mathrm{n}=7(13.2 \%)$.

Concerning Colleges, 14 participants $(26.4 \%)$ were from medicine, 5 (9.4\%) from nursing, 13 (24.5\%) from pharmacy, $7(13.2 \%)$ from dentistry, $13(24.5 \%)$ from applied science, and only 1 participant (1.9\%) was from emergency medical service. With respect to professions, 10 participants were physicians (18.9\%), 5 participants were nurses $(9.4 \%)$, 11 participants were pharmacists $(20.4 \%), 6$ participants were dentists $(11.3 \%)$, and 21 participants $(39.6 \%)$ work in other professions.

Other demographic results included total years of overall academic experience $(\mathrm{M}=13.51, \mathrm{SD}=5.75)$; total years of clinical experience $(\mathrm{M}=11.17, \mathrm{SD}=4.59)$; total years of academic experience at $\mathrm{KSU}(\mathrm{M}=12.47, \mathrm{SD}=5.78)$. Thirty participants (56.4\%) were currently involved in clinical studies, 22 participants (44.1\%) had previous exposure to interprofessional education before, 14 (39\%) attended IP sessions training outside $\mathrm{KSU}$, and the remaining 9 participants $(39.1 \%)$ attended IPE\&P sessions training workshop at KSU. A demographic summary is outlined in Table 1.

\section{Interdisciplinary Education Perception Scale}

The results showed that in terms of attitudes towards IPE\&P, the participants showed positive attitudes towards interprofessional cooperation, including competency and autonomy, the need for cooperation, and the perception of actual cooperation, both pre- and post-workshop. Concerning changes in attitudes of teaching staff towards interprofessional cooperation as a result of the workshop, the analysis showed a statistically significant difference in subscale 1 (competency and autonomy) Mean=23.32 (SD $=5.34)$ versus Mean=24.38 $(\mathrm{SD}=3.61) p=0.03$, where a range from 6 to 30 was possible. In contrast, a statistically significant effect was not produced for either 
Table I Participants' Demography

\begin{tabular}{|l|l|l|l|}
\hline Variables & Descriptor & N & $\begin{array}{l}\text { Percentage } \\
\%\end{array}$ \\
\hline Gender & Male & 30 & $56.6 \%$ \\
& Female & 23 & $43.4 \%$ \\
\hline College & Medicine & 14 & $26.4 \%$ \\
& Nursing & 5 & $9.4 \%$ \\
& Pharmacy & 13 & $24.5 \%$ \\
& Dentistry & 7 & $13.2 \%$ \\
& Applied Medical Science & 13 & $24.5 \%$ \\
& Emergency Medical & 1 & $1.9 \%$ \\
& Services & & \\
\hline Profession & Physician & 10 & 18.9 \\
& Nurse & 5 & 9.4 \\
& Pharmacist & 11 & 20.8 \\
& Dentist & 6 & 11.3 \\
& Others & 21 & 39.6 \\
\hline Academic & Assistant Professor & 35 & $66 \%$ \\
& Associate Professor & 7 & $13.2 \%$ \\
& Professor & 11 & $20.8 \%$ \\
\hline & & & \\
& & &
\end{tabular}

subscale two (perceived need for cooperation) $(p=1.0)$ or subscale three (perception of actual cooperation) between the pre- and post-workshop $(p=0.43)$. Full results can be seen in Table 2 below.

\section{Discussion}

There is now global acknowledgement that both IPC and IPE can improve health outcomes, although both are relatively new in KSA. This study involved teaching staff from across several professions, including medicine, nursing, pharmacy, dentistry, applied medical science and emergency medical services. Previous studies of interprofessional education have also involved diverse disciplines or professions. Giodano ${ }^{23}$ suggests that there is potential

Table 2 Paired Sample Statistics of PRE and POST Workshop IEPS Data

\begin{tabular}{|l|l|l|l|l|}
\hline Factor & Pair & N & Mean & $\begin{array}{l}\text { Std. } \\
\text { Deviation }\end{array}$ \\
\hline Competency and Autonomy & PRE & 53 & 23.32 & 4.54 \\
& POST & 53 & 24.38 & 3.61 \\
Perceived need for & PRE & 53 & 9.96 & 1.78 \\
Cooperation & POST & 53 & 9.96 & 1.64 \\
Perception of Actual & PRE & 51 & 23.55 & 5.28 \\
Cooperation & POST & 51 & 24.02 & 4.68 \\
\hline
\end{tabular}

for close working relationships between teaching staff if they have positive perceptions about IPE and IPC and reflect their practice-based experiences. Although this study focussed on attitudes of teaching staff, there are also some studies in SA that measure the attitudes of students towards IPC. ${ }^{27,28}$ Compared to the number of studies in western countries; however, studies measuring attitudes towards IPE and IPC in SA are few. ${ }^{25}$

The key finding of this study was that the 53-teaching staff from Health Colleges across King Saud University showed a positive attitude towards interprofessional cooperation. This is a pleasing finding given the assertion of Fallatah ${ }^{4}$ that to implement the Saudi 2030 Vision for healthcare; there would need to be a commitment to promote IPE at every level. Barr ${ }^{14}$ also highlights that IPE is "no more effective than its teachers" and emphasizes the importance of teacher competency, commitment, preparation and support. Although we found several studies on the attitudes that faculty or teachers hold regarding IPE, ${ }^{17,22,23,29-32}$ research in this area is still limited. ${ }^{17}$ Of the limited studies we found on teachers' attitudes, only one used the IEPS, and this was the 18 -item version. ${ }^{23}$ Mean factor scores for teaching staff appeared lower in this study than ours. This study adds to the body of knowledge of faculty perceptions of IPE as well as the use of 12-item IEPS as a tool for exploring faculty perceptions.

The increase in positive attitudes about competency and autonomy as a result of the interprofessional development workshop is also promising. Limited attention to faculty development in IPE has been noted by Grymonpre $^{33}$ and this workshop is one small positive step in the wider-system change required for successful IPE. It also lays the foundation for further development workshops. The study also showed that there was no change in participants' perceived need for cooperation or perception of actual cooperation. This is not surprising given the mean scores which suggest that participants already have positive attitudes, where the workshop may have given them deeper insights (competency and autonomy) about IPE, but not changed their already positive attitude towards IPE - which would logically suggest already seeing a need for cooperation. It can also be deduced that teaching staff that attended the workshop attended it because they already saw a need for IPE or engaged in it already. Why other teaching staff did not participate was not explored in this study. 
It is difficult to compare our results with other studies as the IEPS in existing studies have used a longer version of this scale (ie, 18-item), except for a study by Williams et $\mathrm{al}^{24}$ with students, and only one of these studies was with teaching staff. Williams et $\mathrm{al}^{24}$ found that for competency and autonomy, the mean scores for students from 5 Australian universities were 23.45, 24.41, 23.28, 24.61 and 24.41 (possible range 5-30). Perceived need for cooperation scores were 9.70, 9.85, 9.56, 9.98 and 9.33 (possible range 2-12); and perception of actual cooperation were 23.90, 24.91, 23.35, 24.63 and 24.16 (possible range 5-30). Also, it is difficult to state why the teaching staff in the study have positive attitudes or what parts of the intervention worked as the IEPS was implemented only before and after the workshop. Further studies could incorporate a more in-depth qualitative component to tease out these aspects.

This research and other previous research in Saudi Arabia are good starting points to achieve the Saudi 2020 Vision for healthcare; however, it is recommended that more systemic and larger-scale research be carried out. There are some examples of this from other countries, for example, Williams and Teese $^{34}$ conducted a crossinstitutional analysis of paramedic students' attitudes towards IPC providing the first multi-institutional normative data for paramedic students for the IEPS within Australia. Achieving the vision will require IPE and IPC applied across universities, as well as within universities [ie, students and teachers], with support from policy through to practice. Along with the support of teaching staff across health science schools, Fallatah ${ }^{4}$ noted the support also needed by the government, university governors, and administrative and financial support. The World Health Organisation (WHO) provides a "Framework for Action on Interprofessional Education and Collaborative Practice" for policy-makers to implement IPE and IPC within their current context, in recognition that the mechanisms that shape these are not the same in all health systems, and that health leadership is required at local and regional levels to achieve the health-related Millennium Development Goals. ${ }^{1}$

Although the IEPS proved a useful tool to assess attitudes towards IPE, it would have been useful if additional information was collected during this study, particularly the barriers, as well as enablers, to IPE that the teaching staff experienced or could anticipate. Most other studies we found looked at attitudes, as well as other collected additional data, particularly barriers. Many barriers to the implementation of IPE curricula have been reported. El-Awaisi et $\mathrm{al}^{15}$ explain that IPE is more complicated than merely bringing students from different professions together and includes having a good understanding of different professional histories and cultures, and being able to create robust, relevant and meaningful learning experiences and assessing short-term learning goals with long-term practice goals. Although the IEPS suited the purpose for this study, McFadyen et $\mathrm{al}^{21}$ suggested that it is essential that researchers within educational and clinical practice settings have several instruments, including the IEPS, which measure the associated complexities and different aspects of IPC.

Finally, it is suggested that further studies focus on success factors and enablers of IPC and IPE, and not just barriers. In the literature, other barriers include scheduling, space and time for training, matching levels of learners, faculty development, and institutional buy-in; ${ }^{16}$ absent or tepid institutional leadership; difficulties in forming collaborative partnerships; siloed curricula and calendars; and a faculty with limited insight into each other's professions, poorly versed in the knowledge, skills, and attitudes required of collaborative practice, and uncomfortable teaching across professions; ${ }^{8}$ and dealing with non-cohort groups that vary in many attributes such as background, college, specialty, culture, nationality, age, seniority, administrative role and motive in one setting. ${ }^{13}$ Fallatah $^{4}$ has highlighted the challenges faced in KSA, including a lack of specialists in IPE, inflexible curricula, and the lack of skills to teach in IPE-focused curriculum. A 2014 publication of a literature review found 1570 reports about barriers to IPE but only 18 reports about factors that enable universities to deal with them successfully. ${ }^{8}$

\section{Limitations}

In terms of study limitations, this was only a small pilot study representing 53 faculty members at one University. As such, the results are most useful to this University, particularly for developing a strategy for IPC and IPE going forward. Caution is needed when interpreting the results given the lack of external validity.

\section{Conclusions}

Interprofessional collaboration across the Health Colleges is an essential component of IPE, just as IPE is an integral component of interprofessional collaborative practice. The importance of teaching staff modelling positive, proactive behaviour towards IPE to students cannot be overstated, and this highlights the importance of institutional support - and taking a systemic approach to IPE. The findings provided 
a baseline, as well as an incentive, for further development in IPE, from policy through to practice, across the Health Colleges. Further research is needed on tools for measuring IPC across university hierarchies and disciplines, as well as on enablers of IPE (and not just barriers). Teaching staff in the Health Colleges at King Saud University have a positive attitude towards IPE, giving us confidence that we are on the right path towards achieving the Saudi 2030 Vision for healthcare.

\section{Acknowledgments}

The authors extend their appreciation to the Deanship of Scientific Research at King Saud University for funding this work through research group no. RG-1440-105.

\section{Disclosure}

The authors report no conflicts of interest in this work.

\section{References}

1. WHO. Framework for Action on Interprofessional Education \& Collaborative Practice (WHO/HRH/HPN/10.3). 2010.

2. Barr H, Koppel I, Reeves S, Hammick M, Freeth D. Effective Interprofessional Education: Argument, Assumption \& Evidence. Oxford: Blackwell; 2005.

3. Lawrence JC, Knol LL, Clem J, de La OR, Henson CS, Streiffer RH. Integration of interprofessional education (IPE) core competencies into health care education: IPE meets culinary medicine. $J$ Nutr Educ Behav. 2019;51(4):510-512. doi:10.1016/j.jneb.2019.01.013

4. Fallatah H. Introducing inter-professional education in curricula of Saudi health science schools: an educational projection of Saudi vision 2030. J Taibah Univ Sci. 2016;11(6):520-525. doi:10.1016/j. jtumed.2016.10.008

5. Judge MP, Polifroni EC, Maruca AT, Hobson ME, Leschak A, Zakewicz H. Evaluation of students' receptiveness and response to an interprofessional learning activity across health care disciplines: an approach toward team development in healthcare. Int J Nurs Stud. 2015;2(1):93-98. doi:10.1016/j.jijnss.2015.01.003

6. Williams B, Lynch M, Olaussen A, Lachmann H, Kalén S, Ponzer S. Translation and psychometric evaluation of the Swedish version of the interdisciplinary education perception scale. $J$ Interprof Care. 2018;32(1):63-68. doi:10.1080/13561820.2017.1381077

7. Bonello M, Morris J, Muscat N. The role of national culture in shaping health workforce collaboration: lessons learned from a case study on attitudes to interprofessional education in Malta. Health Policy (New York). 2018;122(10):1063. doi:10.1016/j.healthpol.2018.06.013

8. Mladenovic J, Tilden VP. Strategies for overcoming barriers to IPE at a health sciences university. J Interprof Educ Pract. 2017;8:10-13. doi:10.1016/j.xjep.2017.05.002

9. Barr H. Responding as interprofessional educators to the WHO challenge. J Taibah Univ Sci. 2016b;11(6):505-509. doi:10.1016/j. jtumed.2016.08.004

10. Thistlethwaite JE. Interprofessional education: implications and development for medical education. Educ Med. 2015;16(1):68-73. doi:10.1016/j.edumed.2015.04.007

11. Khan TM, Madu Emeka P, Aljadhey H, Haseeb A. Study investigating pharmacy students' interprofessional perceptions toward the pharmacy profession in Saudi Arabia. Curr Pharm Teach Learn. 2015;7(1):62-69. doi:10.1016/j.cptl.2014.09.019
12. Cant R, Leech M, Hood K. Factors affecting Australian medical students' attitudes to interprofessional education; validity of the readiness for inter-professional learning scale-med. $J$ Interprof Educ Pract. 2015;1(3):90-96. doi:10.1016/j.xjep.2015.10.002

13. Al-Sheikh $\mathrm{MH}$. Interprofessional faculty development program: ESHPE model. $J$ Interprof Educ Pract. 2018;12:29-32. doi:10.1016/j.xjep.2018.05.003

14. Barr H. Promoting interprofessional education. J Taibah Univ Sci. 2016a;11(6):503-504. doi:10.1016/j.jtumed.2016.10.009

15. El-Awaisi A, Anderson E, Barr H, Wilby KJ, Wilbur K, Bainbridge L. Important steps for introducing interprofessional education into health professional education. J Taibah Univ Sci. 2016;11 (6):546-551. doi:10.1016/j.jtumed.2016.09.004

16. Chen AK, Dennehy C, Fitzsimmons A, et al. Teaching interprofessional collaborative care skills using a blended learning approach J Interprof Educ Pract. 2017;8:86-90. doi:10.1016/j.xjep.2017.07.002

17. Dallaghan G, Hoffman E, Lyden E, Bevil C. Faculty attitudes about interprofessional education. Med Educ Online. 2016;21(1):1-6. doi:10.3402/meo.v21.32065

18. Hammick M, Freeth D, Koppel I, Reeves S, Barr H. A best evidence systematic review of interprofessional education: BEME guide no. 9. Med Teach. 2007;29(8):735. doi:10.1080/01421590701682576

19. Milutinovic D, Lovric R, Simin D. Interprofessional education and collaborative practice: psychometric analysis of the readiness for interprofessional learning scale in undergraduate Serbian healthcare student context. Nurse Educ Today. 2018;65:74. doi:10.1016/j. nedt.2018.03.002

20. Vaughan B. Measurement properties of the interdisciplinary education perception scale in an Australian allied health student cohort. Health Prof Educ. 2019;5(3):275-280. doi:10.1016/j.hpe.2018.07.005

21. McFadyen AK, Maclaren WM, Webster VS. The interdisciplinary education perception scale (IEPS): an alternative remodelled sub-scale structure and its reliability. $J$ Interprof Care. 2007;21 (4):433-443. doi:10.1080/13561820701352531

22. Curran VR, Sharpe D, Forristall J. Attitudes of health sciences faculty members towards interprofessional teamwork and education. Med Edu. 2007;41(9):892-896. doi:10.1111/j.13652923.2007.02823.x

23. Giordano C, Umland E, Lyons K. Attitudes of faculty and students in medicine and the health professions toward interprofessional education. $J$ Allied Health. 2012;41(1):21-25.

24. Williams B, Boyle M, Brightwell R, et al. A cross-sectional study of paramedics' readiness for interprofessional learning and cooperation: results from five universities. Nurse Educ Today. 2013;33 (11):1369-1375. doi:10.1016/j.nedt.2012.06.021

25. Williams B, Webb V. Examining the measurement properties of the interdisciplinary education perception scale (IEPS) in paramedic education. Nurse Educ Today. 2013;33(9):981-985. doi:10.1016/j. nedt.2012.10.015

26. Luecht R, Madsen M, Taugher M, Petterson B. Assessing professional perceptions: design and validation of an interdisciplinary education perception scale. J Allied Health. 1990;19(2):181-191.

27. El-Zubeir M, Rizk DEE, Al-Khalil RK. Are senior UAE medical and nursing students ready for interprofessional learning? Validating the RIPL scale in a Middle Eastern context. J Interprof Care. 2006;20 (6):619-632. doi:10.1080/13561820600895952

28. Fallatah H, Jabbad R, Fallatah H. Interprofessional education as a need: the perception of medical, nursing students and graduates of medical college at King Abdulaziz University. Creative Educ. 2015;6 (02):248-254. doi:10.4236/ce.2015.62023

29. Bennett PN, Gum L, Lindeman I, et al. Faculty perceptions of interprofessional education. Nurse Educ Today. 2011;31(6):571-576. doi:10.1016/j.nedt.2010.09.008

30. Gary JC, Gosselin K, Bentley R. Health science center faculty attitudes towards interprofessional education and teamwork. $J$ Interprof Care. 2018;32(2):231-234. doi:10.1080/13561820.2017.1376626 
31. Loversidge J, Demb A. Faculty perceptions of key factors in interprofessional education. $J$ Interprof Care. 2015;29(4):298-304. doi:10.3109/13561820.2014.991912

32. Vernon MM, Moore NM, Cummins L-A, et al. Respiratory therapy faculty knowledge of and attitudes toward interprofessional education. Respir Care. 2017;62(7):873. doi:10.4187/respcare. 05034
33. Grymonpre RE. Faculty development in interprofessional education (IPE): reflections from an IPE coordinator. J Taibah Univ Sci. 2016;11(6):510-519. doi:10.1016/j.jtumed.2016.10.006

34. Williams B, Teese D. A cross-institutional analysis of Australian undergraduate paramedic students' attitudes towards interprofessional collaboration. J Interprof Care. 2016;30(1):97. doi:10.3109/ 13561820.2015.1070132

\section{Publish your work in this journal}

The Journal of Multidisciplinary Healthcare is an international, peerreviewed open-access journal that aims to represent and publish research in healthcare areas delivered by practitioners of different disciplines. This includes studies and reviews conducted by multidisciplinary teams as well as research which evaluates the results or conduct of such teams or healthcare processes in general. The journal covers a very wide range of areas and welcomes submissions from practitioners at all levels, from all over the world. The manuscript management system is completely online and includes a very quick and fair peer-review system. Visit http://www.dovepress.com/testimonials. php to read real quotes from published authors. 